\title{
Comparative Gene Expression Profiling in Gultured Cell Lines after Treatment with Wasabi-derived Isothiocyanates
}

\author{
Phoebe Trio ${ }^{1,3, *}$, Atsuyoshi Kawahara ${ }^{2}$, Satoru Fujisaki ${ }^{2}$, De-Xing Hou ${ }^{2,3}$ \\ ${ }^{1}$ Chemistry Department, College of Science, De La Salle University - Manila, 2401 Taft Avenue, Manila \\ ${ }^{2}$ Department of Biochemical Sciences and Biotechnology, Faculty of Agriculture, Kagoshima University, \\ Korimoto 1-21-24, Kagoshima 890-0065, Japan \\ ${ }^{3}$ United Graduate School of Agricultural Sciences, Kagoshima University, Korimoto 1-21-24, Kagoshima 890- \\ 0065, Japan
}

\begin{abstract}
Isothiocyanate (ITC) is a small but reactive organo-sulfur containing group of compound present in cruciferous vegetables. Compendium of evidence indicated that ITCs exhibit multiple biological activities, but the exact molecular mechanisms are not yet clear. Therefore, this study was designed to compare the genome-wide gene expression profiles in hepatic and neuron cells following wasabi-derived ITCs treatment using microarray technology. Cells were treated with wasabi-derived ITCs, sulforaphane (SFN), 6-(methylsulfinyl)hexyl isothiocyanate (6-MSITC) and 6-(methylthio)hexyl isothiocyanate (6-MTITC), for $9 \mathrm{~h}$ and was followed by DNA microarray analyses using HG-U133 plus 2.0 oligonucleotide array. Selected gene products were confirmed by real-time PCR, and functional subsets of genes and biologically significant network were identified using Ingenuity Pathway Analysis. Results showed that 6-MTITC was the most potent inducer of gene expressions changes in HepG2 cells, whereas 6-MSITC was the most effective inducer in IMR-32 cells. Despite this cell-type response discrepancies, 6-MSITC came up as the strongest inducer of antioxidant-associated genes, via the regulation of the Nrf2-mediated pathway. These results combined with the varying induction level data of other Wasabi-derived ITCs form the basis for further studies to assess the possible therapeutic effect of combined Wasabi-derived ITCs treatment. Altogether, this study provided comprehensive information on how structural differences of Wasabi-derived ITCs contribute to its efficacy and impact specific targets.
\end{abstract}

Keywords: gene expression profiling; isothiocyanates; Japanese wasabi

\section{INTRODUCTION}

Isothiocyanate (ITC) is a small but reactive organosulfur containing molecule present in most cruciferous vegetables. Ever since the first report of its antitumor activity in vivo, ITCs continue to attract the attention of many researchers (Sidransky et al., 1966). Recent studies showed that ITC sulforaphane (SFN) can network with numerous signaling pathways associated with carcinogenesis and modify epigenetic events (Myzak et al., 2006; Atwell et al., 2015). The compound can also upregulate antioxidant-related enzymes and downregulate inflammatory mediators and cytokines in response to oxidative stress ( $\mathrm{Ye}$ et al., 2013). In addition, SFN is associated with the prevention of neurodegenerative diseases by inducing cytoprotective proteins (Tarozzi et al., 2013). 
In particular, studies revealed that the presence of high amount of ITCs in Japanese Wasabi contributes to its biological and pharmacological activities. For example, allyl-isothiocyanate (AITC) and 6-(methylsulfinyl) hexyl isothiocyanate (6-MSITC) can strongly inhibit the growth of Staphylococcus aureus, Escherichia coli, and Helicobacter pylori even at a very low concentration (Isshiki \& Tokouka, 1993; Ono et al., 1998; Shin et al., 2004). AITC can also promote weight loss by suppressing adipogenesis or lipogenesis (Kim et al., 2015). Another Wasabi-derived ITC, 6-(methylthio) hexyl isothiocyanate (6-MTITC), showed to inhibit platelet aggregation and promote deaggregation more potent than aspirin (Kumagai et al., 1994). On the other hand, 6-MSITC can prevent lipopolysaccharide (LPS)-induced macrophage activation, arachidonicor adenosine-diphosphate induced platelet activation and tumor cell proliferation in vitro (Okamoto et al., 2013). In murine macrophage, RAW264, 6-MSITC exhibited anti-inflammatory activity by inhibiting cyclooxygenase-2 (COX-2) or interferon- $\gamma(\mathrm{IFN}-\gamma)$, and suppressing iNOS expression via inhibition of Jak2 mediated JNK signaling cascade (Uto et al., 2005a; Uto et al., 2007; Uto et al., 2005b). Genomewide study in mouse macrophage demonstrated the anti-inflammatory function of 6-MSITC via regulation of chemokines, interleukins and interferons (Chen et al., 2010). The extract of Wasabi was also found to enhance adiponectin secretion, and inhibit tumor necrosis factor- $\alpha$ (TNF- $\alpha$ )-stimulated lipolysis and IL- 6 secretion comparable to an anti-diabetic drug, troglitazone (Babish et al., 2010). However, conflicting results were published on its anti-oxidant potency. Studies of Ryu et al. (2007) and Lee (2008) showed that water extract is more potent inhibitor of radicals than alcohol extract. Whereas, Shin et al. (2014) revealed that alcohol extracts had higher radical scavenging activity than the water extract. Irrespective of the contradictory figures, evidence showed that Wasabi possesses antioxidant function. Thus, compendium of evidence suggests that Wasabi cannot only be used as a condiment but can be also utilized for prevention and cure of some diseases. However, limited studies are available dealing with its global-wide mechanisms to exert pharmacological effects.

With the rise of nutrigenomics research, studies that provide molecular biomarkers or gene expression patterns due to the whole diet or individual dietary constituents are now feasible. These studies are of great help to the improvement of a person's wellbeing. However, to attain this purpose, the need of a high throughput functional genomic technique such as microarray is crucial. Microarray technique is a robust and suitable technique for gene-diet interaction research studies for it permits scholars to identify the therapeutic function of a natural food component and at the same time allows us to understand why and how some natural foods may induce varying gene responses. Therefore, this study was designed to compare the global changes in transcript levels and the underlying genes targeted by Wasabi-derived ITCs in hepatic cells with that of neuronal cells using microarray-based technology. Multiple ITC samples with structural variabilities were designed to identify the impact of structural difference towards biological functions of Wasabi-derived ITCs. cDNA microarray is applied to acquire novel information regarding the effect of the particular Wasabi-derived ITCs at the genome-wide level. Furthermore, different cell lines were chosen as in vitro models to demonstrate distinct gene expression patterns in response to ITCs stimulation in cells of hepatic versus neuronal origin. Altogether, the approach of this study would provide novel information how structural differences of Japanese Wasabi-ITCs contribute to its efficacy and affect specific targets.

\section{EXPERIMENTAL}

Chemicals. Sulforaphane (SFN), 6-MSITC and 6-MTITC were isolated from Wasabi and purified (99.3 \%) by gas chromatography (Hou et al., 2000). All the three isothiocyanates were dissolved in DMSO for cell culture experiments.

Cell Culture. Human neuroblastoma IMR-32 cells (cell no. TKG0207) and human hepatoblastoma Hep2G cells (cell no. TKG0205) were both obtained from Riken Bioresource Center Cell Bank (Ibaraki, Japan). IMR-32 cells were grown in Eagle's Minimum Essential Medium (EMEM, Nissui, Seiyaku, Tokyo, Japan) supplemented with $1 \% \mathrm{v} / \mathrm{v} 2 \mathrm{mM}$ L-glutamine (Nacalai, Tesque, Kyoto, Japan), 1\% v/v MEM non-essential amino acid solution (NEAA, Nacalai, Tesque, Kyoto, Japan), and $10 \% \mathrm{v} / \mathrm{v}$ fetal bovine serum (FBS, EquitechBio, Kerrville, TX USA) under a humidified 5\% $\mathrm{CO}_{2}$ atmosphere at $37^{\circ} \mathrm{C}$. HepG2 cells were grown in DMEM (Dulbecco's Modified Eagle Medium, Nissui, Seiyaku, Tokyo, Japan) containing 10\% FBS (Equitech-Bio, Kerrville, Texas, USA) under humidified $5 \% \mathrm{CO}_{2}$ atmosphere at $37{ }^{\circ} \mathrm{C}$. Cells were treated with or without ITCs for $12 \mathrm{~h}$, unless otherwise indicated.

\section{RNA Preparation and Microarray Hybridization.} Cells were pre-cultured in $10 \mathrm{~cm}$ dishes for $24 \mathrm{~h}$, followed by $10 \mu \mathrm{M}$ ITCs in $0.2 \% \mathrm{v} / \mathrm{v}$ DMSO treatment for $9 \mathrm{~h}$. Total RNA was extracted using RNeasy Mini Kit (Qiagen ${ }^{\mathrm{Tx}}$, Valencia, CA) following the manufacturer's instructions. RNA integrity was assessed using Agilent 2100 bioanalyzer (Agilent Technologies, Palo Alto, CA, USA). 
400 ng RNA was amplified using Eukaryotic Poly-A RNA control kit (Affymetrix, Santa Clara, CA, USA) and GeneChip ${ }^{\oplus}$ One-cycle cDNA synthesis kit (Affymetrix, Santa Clara, CA, USA), following the manufacturer's protocol to generate cDNA, followed by in vitro transcription and biotin labeling to generate labelled $\mathrm{cRNAs}$ using GeneChip ${ }^{\circledR}$ in vitro transfection kit (Affymetrix, Santa Clara, CA, USA). The fragmented and biotin-labelled cRNAs were hybridized to Human Genome (HG) U133 Plus 2.0 oligonucleotide arrays (GeneChip ${ }^{\circledR}$, Affymetrix, Santa Clara, CA, USA) containing about 54,000 probe sets, followed by washing to remove unbound cRNAs.

Data Analysis. Hybridized fluorescence was scanned using Affymetrix Launcher. Images were processed using GeneSpring GX 10.1 (Agilent Technologies, Palo Alto, CA, USA) for visualization and normalization of each probe set to a common baseline. The untreated cells were used as baseline gene expression and data between arrays were normalized using quantile method. Then normalized data were subjected to log base 2 transformation. The probes fold change greater than 2 and $P<0.05$ were defined as differentially expressed genes. Gene products of fold change greater than two were further analyzed using Gene Ontology software (www.geneontology. org) for biological processes, molecular functions, and signaling pathways.

Statistical analysis was performed by analysis of variance (ANOVA) and $P$ value of $<0.05$ to determine statistical difference between the array data of the control and treated samples. Fisher's right tail $t$-test was used to determine that the canonical pathway assigned to a given data set is not a product of chance alone. $P$ value of $<0.05$ was considered statistically significant.

Pathway Analysis and Network Generation. Pathway and global functional analysis were performed using Ingenuity Pathway Analysis (IPA, Ingenuity ${ }^{\oplus}$ Systems, www.ingenuity.com). A data set containing gene accession numbers and its corresponding fold change were uploaded into the software and was mapped out using the Ingenuity Pathways Knowledge Base (IPKB). IPKB analysis generated the biological functions as well as pathways from the IPA library that is most significant to the data set. Genes from the data sets associated with biological functions or with the canonical pathway with $P$ value smaller than 0.005 were used to map out molecular networks. Resulting networks were ranked based on the scores generated from Fisher exact test to indicate the probability of the biological function and/or canonical pathway was not due to chance alone.
Reverse Transcription and Real-time PCR. Significantly up-regulated gene expressions from microarray experiment were validated using realtime PCR. Primers used for each specific gene (Table 1), designed based on the NCBI sequence database using Primer3 v.0.4.0. RNA, was reverse-transcribed into cDNA using Oligo dT and M-MuLV RNase in a GeneAmp PCR System 2400 following the described reaction cycles. Denaturation was performed at 25 ${ }^{\circ} \mathrm{C}$ for $10 \mathrm{~min}$, followed by annealing and extension at $37^{\circ} \mathrm{C}$ for $30 \mathrm{~min}$ and $85^{\circ} \mathrm{C}$ for $5 \mathrm{~min}$, respectively. Reverse transcription and real-time PCR (RT-PCR) were performed using DyNAmo ${ }^{\mathrm{TM}} \mathrm{SYBR}^{\circledR}$ Green 2-Step qRT-PCR Kit (Finnzymes Oy, Espoo, Finland) following the manufacturer's procedure. Quantitative PCR was done with a standard curve using Rotor-Gene-3000AKAA (Corbett Research) in triplicate. The thermal cycling conditions applied were the following: holding at $95^{\circ} \mathrm{C}$ for $1 \mathrm{~min}, 50$ 60 cycles at $95^{\circ} \mathrm{C}$ for $15 \mathrm{sec}$, corresponding $\mathrm{Tm}$ at $30 \mathrm{sec}, 72^{\circ} \mathrm{C}$ for $30 \mathrm{sec}$ and melting at $72-95^{\circ} \mathrm{C}$ for $45 \mathrm{sec}$. The results were represented by the relative expression level normalized with control cells.

Table 1. Primers Used for Real-time PCR.

\begin{tabular}{|c|c|c|c|}
\hline Genes & Direction & Sequences & $\mathrm{T}_{\mathrm{m}}\left({ }^{\circ} \mathrm{C}\right)$ \\
\hline \multirow{2}{*}{$A K R 1 C 1$} & $\mathrm{Fw}$ & $\begin{array}{l}\text { ATC CCT CCG AGA AGA } \\
\text { ACC AT }\end{array}$ & \multirow{2}{*}{59} \\
\hline & $\mathrm{Re}$ & $\begin{array}{l}\text { ACA CCT GCA CGT TCT } \\
\text { GTC TG }\end{array}$ & \\
\hline \multirow{2}{*}{ AKR1C3 } & $\mathrm{Fw}_{\mathrm{w}}$ & $\begin{array}{l}\text { AAG TAA AGC TTT GGA } \\
\text { GGT CAC A }\end{array}$ & \multirow{2}{*}{59} \\
\hline & $\mathrm{Re}$ & $\begin{array}{l}\text { GGA CCA ACT CTG GTC } \\
\text { GAT GAA }\end{array}$ & \\
\hline \multirow{2}{*}{$G C L M$} & $\mathrm{Fw}_{\mathrm{w}}$ & $\begin{array}{l}\text { GGG AAC CTG CTG AAC } \\
\text { TGG }\end{array}$ & \multirow{2}{*}{61} \\
\hline & $\mathrm{Re}$ & $\begin{array}{l}\text { GCA TGA GAT ACA GTG } \\
\text { CAT TCC }\end{array}$ & \\
\hline \multirow{2}{*}{ NQOI } & $\mathrm{Fw}_{\mathrm{w}}$ & $\begin{array}{l}\text { CTG GTT TGA GCG AGT } \\
\text { GTT CA }\end{array}$ & \multirow{2}{*}{60} \\
\hline & $\mathrm{Re}$ & $\begin{array}{l}\text { TTC CAT CCT TCC AGG } \\
\text { ATT TG }\end{array}$ & \\
\hline \multirow{2}{*}{ TXNRDI } & $\mathrm{Fw}_{\mathrm{w}}$ & $\begin{array}{l}\text { ATC AGG AGG GCA GAC } \\
\text { TTC AA }\end{array}$ & \multirow{2}{*}{61} \\
\hline & $\mathrm{Re}$ & $\begin{array}{l}\text { CCC ACA TTC ACA CAT } \\
\text { GTT CC }\end{array}$ & \\
\hline
\end{tabular}

\section{RESULTS AND DISCUSSIONS}

ITCs have been widely reported to exhibit different protective effects such as anti-proliferative, neuroprotective, anti-inflammatory and anticancer activities (Chaudhuri et al., 2007; Tarrozi et al., 2013; Sun et al., 2015; Chung et al., 2015). However, microarray-based method of determining Wasabi-derived ITCs biological effects is rare. Thus, this is the first study to perform simultaneous 
microarray-based gene transcription profiling to contrast the genome wide-gene expression changes associated with Wasabi SFN, 6-MSITC and 6-MTITC stimulation in HepG2 cells with that of IMR-32 cells.

\section{Effect of Wasabi-derived ITC on Gene Expression} Profile Changes of Cells. In Figure 1, 6-MTITC displayed as the strongest inducer of gene expression changes in HepG2 cells as indicated by the total number of differentially altered gene expressions. Further assessment of the significantly up- and downregulated genes demonstrated that ITCs caused upregulation of most of the genes. Whereas, in IMR-32 cells, 6-MSITC had greater influence than 6-MTITC and SFN in the gene expression regulation. Comparison of the direction of regulation showed that 6-MSITC and 6-MTITC had stronger effect towards the downregulation of IMR-32 genes. Structural evaluation of the two ITCs as displayed in Figure 2 suggests that the carbon chain backbone linking the ITC group and the methyl sulfinyl group may play a role in the potency of 6-MSITC and 6-MTITC as inducer of gene expression changes in the two cell lines. Both 6-MSITC and 6-MTITC have six methylene groups linking the methyl sulfur and isothiocyanate group as compared with SFN that has only four methylene groups between the methyl sulfur and isothiocyanate group. However, no direct relationship can be established on the effect of sulfur substituent attached to methyl group of Wasabi-derived ITCs. This observation seemed to be inconsistent with the published studies that change of the oxidation state of the sulfur atom attached to the methyl group from sulfide to sulfoxide enhanced the potency of alkyl ITCs (Zhang et al., 1992; Vasanthi et al., 2009). Yet, it should be noted that the reported studies evaluated structure-bioactivity relationship of different kinds of ITC and not the genome-wide expression effect. This is vital since this is the first report to demonstrate global gene expression of

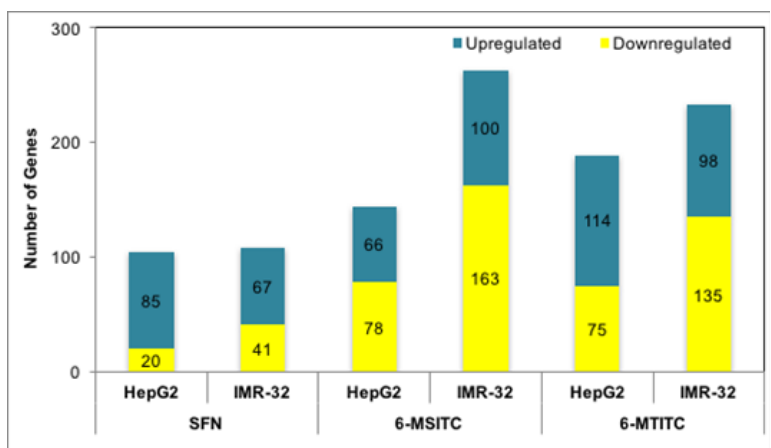

Figure 1. Comparative Total Number of Genes Regulated by ITCs in HepG2 and IMR-32 Cell Lines using Affymetrix HG UG133 Plus 2.0 Oligonucleotide Arrays Containing 54,000 Probe Sets after Treatment with 10 $\mu M$ of SFN, 6-MSITC and 6-MTITC for 9 hrs.

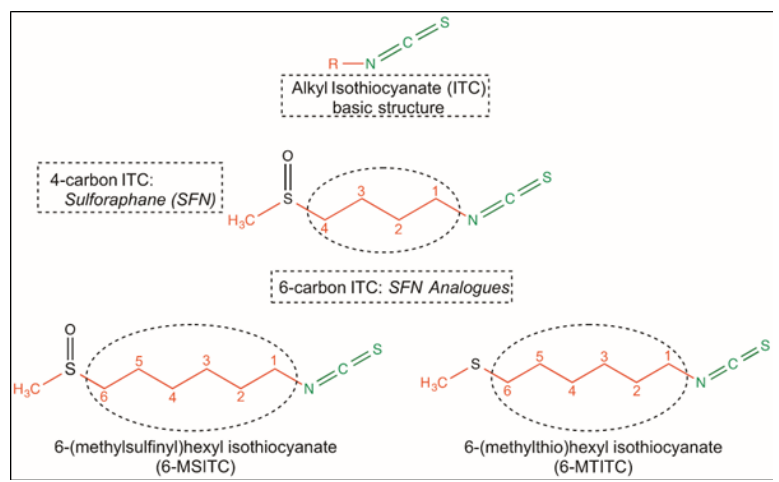

Figure 2. Structure-Gene Expression Profile Relationship of Wasabi-derived ITCs. The methylene groups bridging the methyl sulfur group and isothiocyanate group showed to be the essential component in the potency of ITCs as regulator of gene expression changes in HepG2 and IMR-32 cell culture models. However, not much difference is observed in the potency of sulfur and sulfoxide groups.

changes induced by SFN and SFN analogues and this inconsistency could be possibly attributed to the cell variation which is in accordance with the results of Trio et al., (2017) and Trio et al., (2016). The previous studies indicated that 6-MTITC is the strongest inducer of gene expression changes in HepG2 cells while 6-MSITC is the strongest inducers in IMR-32 cells. Still, comparing the results of the two studies showed that both 6-MSITC and 6-MTITC exert protective effects via its antioxidant activity through the Nrf2 pathway.

Influence of Cell Type Variations on Gene Expression Profiles following Treatment of Wasabi-derived ITC. HepG2 and IMR-32 cells

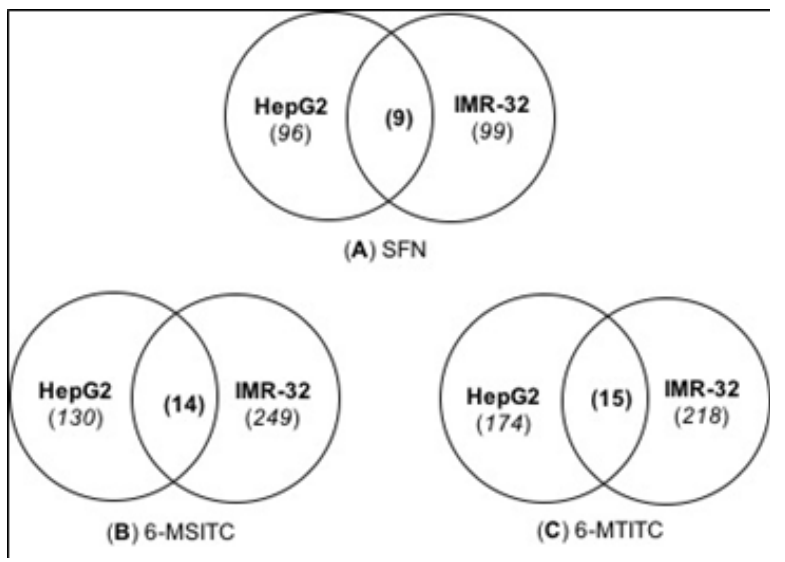

Figure 3. Comparative Venn Diagram Representation of Hep G2 and IMR-32 Cells Gene Expression Profile from Microarray Data in Response to Wasabi-derived ITCS, (A) SFN, (B) 6-MSITC and (C) 6-MTITC Stimulations. Common genes between two cell lines is represented by overlapping circles. Unique genes between two cell lines is displayed in non-overlapping part of the circle. 
were derived from different tissues suggesting that genetic variations exist within the two cell lines. However, studies regarding the effect of genetic variation on gene expression levels across tissues remained elusive. Here, we found large discrepancies in the gene expression profiles of the two cell models following ITC treatments; thus, the effect of cell type variation was taken into account.

As a whole, IMR-32 ITC-treated cells had higher total number of differentially altered genes than HepG2 ITC-treated cells (Figure 1). In 6-MSITC treatment, the IMR-32 cells gene expression profile was $83 \%$ greater than HepG2 cells in terms of the total number of differentially altered genes. A similar relationship is also observed between HepG2 cells and IMR-32 cells treated with 6-MTITC. Surprisingly, HepG2 and IMR-32 produced no significant variation (1\% difference) in response to SFN treatment. However, SFN was found to trigger upregulation of most genes regardless of what cell type used. These observations suggest that the type of cell influenced the number of genes turned on or shut off in response to ITCs. Different kinds of cells have different specialized roles to perform (Mazzarello, 1999) but the knowledge of cellular diversity still remains incomplete and have been subjected to continuous debate. HepG2 is a hepatoblastomaderived cell line commonly used for the study of liver metabolism and development, chemocarcinogenesis, mutagenesis and hepatotoxicity. HepG2 genetic profile revealed losses of the chromosome $4 \mathrm{q} 3$ region and other typical hepatoblastoma chromosomal abnormalities, which includes trisomies 2 and 20 (Lopez-Terrada et al., 2009). In contrast, IMR-32 is of human origin and mimics large projection of neurons of the cerebral cortex. It has been generally used in studies related to the stability of the amyloid precursor protein (Lahiri, 1993).

Furthermore, gene expressions pattern analysis showed that the differentially expressed genes common between HepG2 and IMR-32 cells in response to ITCs stimulation were mostly associated to antioxidant-related genes (Figures 3AC). Among the antioxidant associated genes were AKR1C1, DNAJB4, GCLM, HMO1, HSPA1A/II HSPA1B, OSGIN1, SQSTM1, and SRXN1. These genes code for metabolizing enzymes, detoxifying proteins and antioxidant protein. Fold changes of the selected antioxidant-related genes obtained via microarray analyses demonstrated substantial agreement with the fold change values determined via real-time $\mathrm{PCR}$, thereby confirming the microarray data (Figures 4A-B). These genes were selected based also on the significantly regulated pathway and differentially expressed transcription factors. Detailed investigation of the significantly
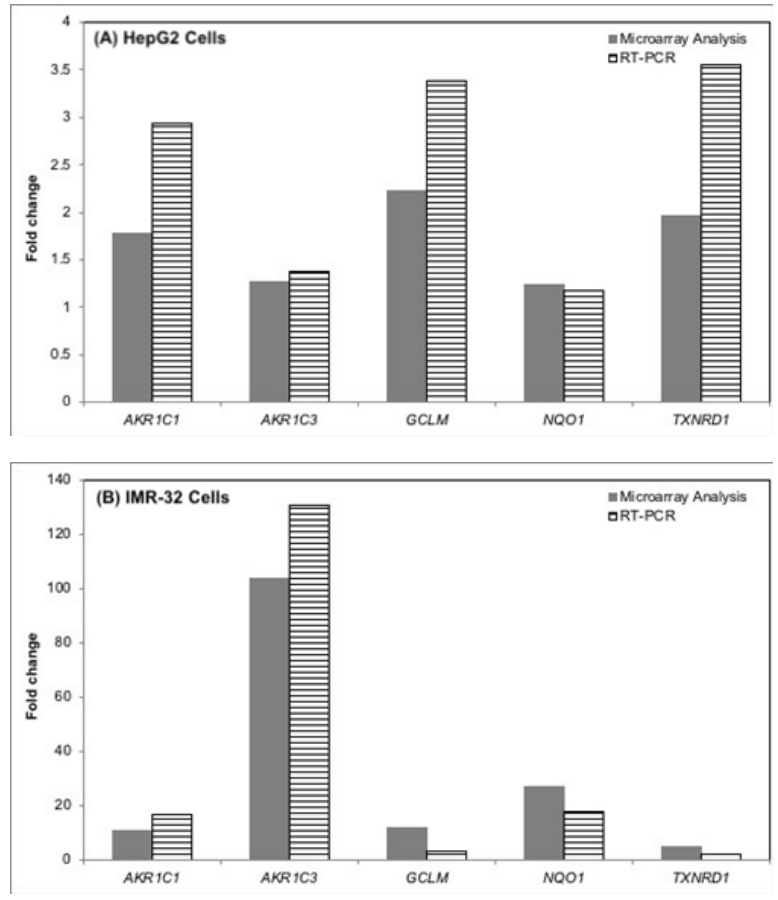

Figure 4. Validation of differentially expressed genes in 6-MSITC-treated (A) HepG2 cells and (B) IMR32 cells from DNA microarray analyses by real-time PCR. DNA microarray results were compared to realtime PCR results for selected genes. Real-time PCR was performed using DyNAmo ${ }^{\text {TM }} S Y B R^{\oplus}$ Green 2-Step qRT-PCR Kit as described in "Section 2". Fold changes represented the ratio between the treated samples values to that of the untreated samples. Expression changes are depicted as fold change (y-axis). Gene symbols are shown in $x$-axis.

modulated pathway showed that the transcription factor involved is Nrf2. Nrf2 is the major controller of most of the antioxidant associated genes; thus, it is important to validate the effect of $\mathrm{Nrf2}$ on its downstream genes by taking into account the expression level of these downstream genes (Itoh $e t$ al., 1997; Nguyen et al., 2000).

GO enrichment analysis revealed that cell proliferation and inflammatory response were distinct to HepG2 in response to ITCs stimulation, while CNS specific function was unique to IMR32 cells (Table 2). ADAMTS1, ADM, CCL14/// CCL15, DAB2, FOSL1, HMOX1, IHH, IL11, JAG1, KITLG, KLF4, PROX1, and TRIB1 were overexpressed genes related to cell proliferation in HepG2. Meanwhile, the overexpressed genes linked with inflammatory response were CCL20, CYP4F11, FOS, LY96, SCYE1, TLR6, and TNFRSF1A. Some of the overexpressed CNSspecific function-related genes in IMR-32 cells were also expressed in HepG2 cells but CCDC50, NGEF, $P D G F C$, and $P R C D$ genes were specific to IMR32 cells. These genes play a role in neuroplasticity, 
Table 2. Comparative Classification of Genes Annotated for Biological Processes Targeted by Wasabiderived ITCs in HepG2 and IMR-32 Cell Lines.

\begin{tabular}{|c|c|c|c|c|c|c|}
\hline \multirow{2}{*}{ Category } & \multicolumn{3}{|c|}{ HepG2 } & \multicolumn{3}{|c|}{ IMR-32 } \\
\hline & SFN & 6-MSITC & 6-MTITC & SFN & 6-MSITC & 6-MTITC \\
\hline Adhesion & 3 & 7 & 9 & 1 & 4 & 4 \\
\hline Apoptosis & 7 & 14 & 17 & 2 & 7 & 5 \\
\hline Autophagy & 0 & 0 & 0 & 1 & 1 & 1 \\
\hline Binding & 0 & 1 & 1 & 5 & 26 & 15 \\
\hline Biogenesis & 2 & 4 & 3 & 0 & 1 & 0 \\
\hline $\begin{array}{l}\text { Catabolic } \\
\text { process }\end{array}$ & 6 & 11 & 12 & 2 & 3 & 3 \\
\hline $\begin{array}{l}\text { Catalytic } \\
\text { activity }\end{array}$ & 0 & 0 & 0 & 0 & 2 & 1 \\
\hline Cell cycle & 2 & 2 & 6 & 0 & 2 & 1 \\
\hline Cell growth & 1 & 2 & 2 & 1 & 1 & 1 \\
\hline $\begin{array}{l}\text { Cell prolifer- } \\
\text { ation }\end{array}$ & 5 & 10 & 12 & 0 & 1 & 2 \\
\hline $\begin{array}{l}\text { CNS specific } \\
\text { function }\end{array}$ & 0 & 0 & 0 & 3 & 10 & 5 \\
\hline DNA repair & 1 & 1 & 1 & 1 & 1 & 1 \\
\hline $\begin{array}{l}\text { Inflammatory } \\
\text { response }\end{array}$ & 4 & 6 & 5 & 0 & 1 & 0 \\
\hline $\begin{array}{l}\text { Metabolic } \\
\text { process }\end{array}$ & 12 & 21 & 23 & 9 & 22 & 17 \\
\hline $\begin{array}{l}\text { Oxidoreduc- } \\
\text { tase activity }\end{array}$ & 4 & 5 & 7 & 10 & 15 & 16 \\
\hline $\begin{array}{l}\text { Response to } \\
\text { stimuli }\end{array}$ & 7 & 7 & 8 & 0 & 2 & 2 \\
\hline $\begin{array}{l}\text { Signal trans- } \\
\text { duction }\end{array}$ & 12 & 33 & 29 & 10 & 22 & 21 \\
\hline Stress response & 6 & 8 & 14 & 5 & 7 & 8 \\
\hline Transcription & 13 & 26 & 36 & 16 & 41 & 36 \\
\hline $\begin{array}{l}\text { Transferase } \\
\text { activity }\end{array}$ & 5 & 12 & 16 & 5 & 10 & 9 \\
\hline Translation & 2 & 4 & 7 & 2 & 2 & 4 \\
\hline Transport & 16 & 33 & 30 & 9 & 20 & 19 \\
\hline
\end{tabular}

*Data figures represent the number of significantly regulated genes per biological processes.

neuro-regeneration and repair, and inflammation in our central nervous system (Lemarchant et al., 2013). With that observation, it can be inferred that genes and processes related to carcinogenesis or tumorigenesis were intrinsically active in HepG2 cell line while genes and processes associated to neuro response function were inherently functional in IMR-32 cell line (Lopez-Terrada et al., 2009; Lahiri, 1993). Neuro-specific genes play a critical role in nervous system by controlling the blood brain barrier homeostasis (Funa and Sasahara, 2014). They also modulate neuronal excitability via ion channels adjustment, and affect synaptic plasticity and function. The extent of activation of the cell proliferation and inflammatory response-associated genes by ITCs in HepG2 cells suggests that it is an ideal cell type for identification of other signal transduction cascade involved in cell proliferation and inflammation. Whereas, the ability of IMR32 cells to actively express genes related to CNS specific function implies that this cell line is suitable for neuroprotective mechanism studies. 
(A)

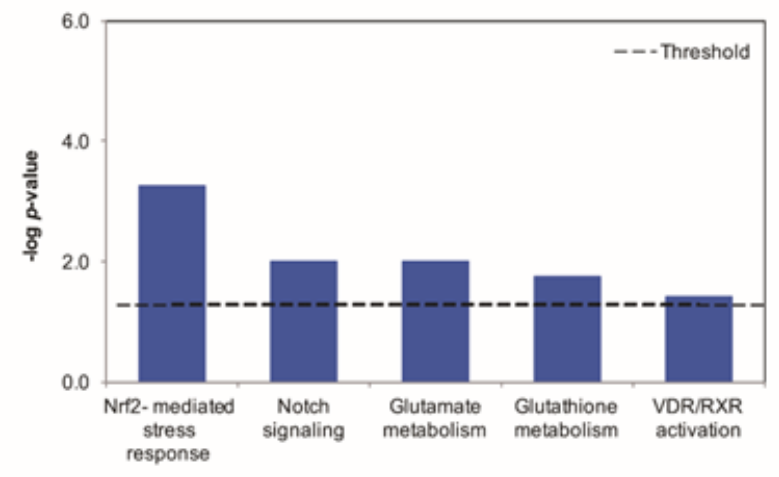

(B)

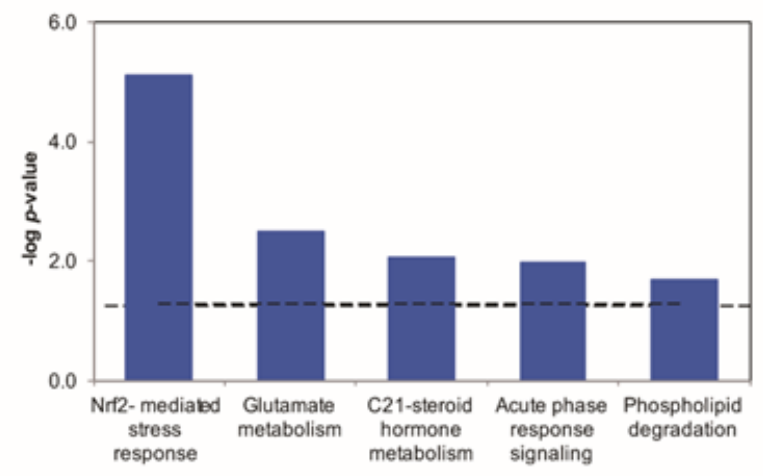

(C)

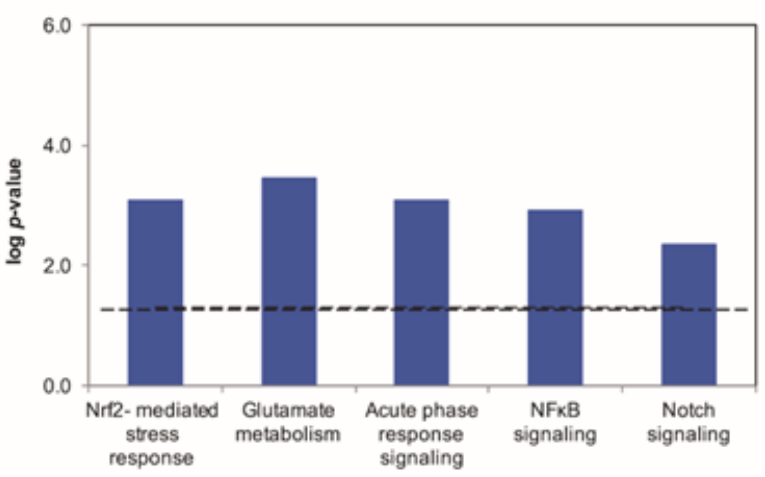

(D)

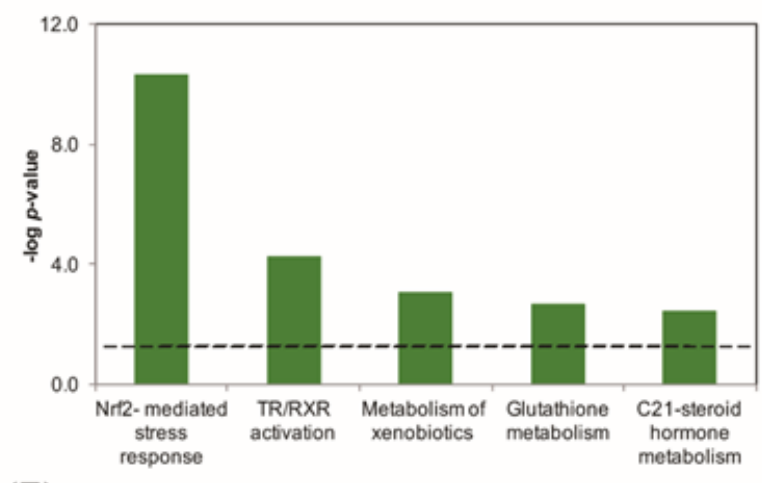

$(\mathrm{E})$

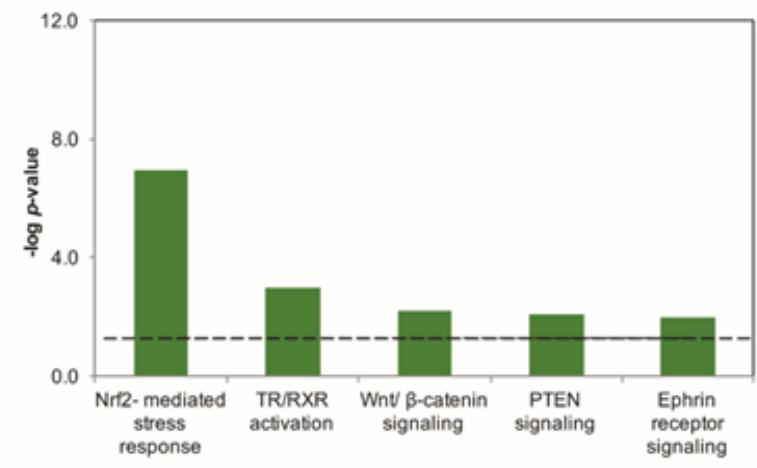

$(\mathbf{F})$

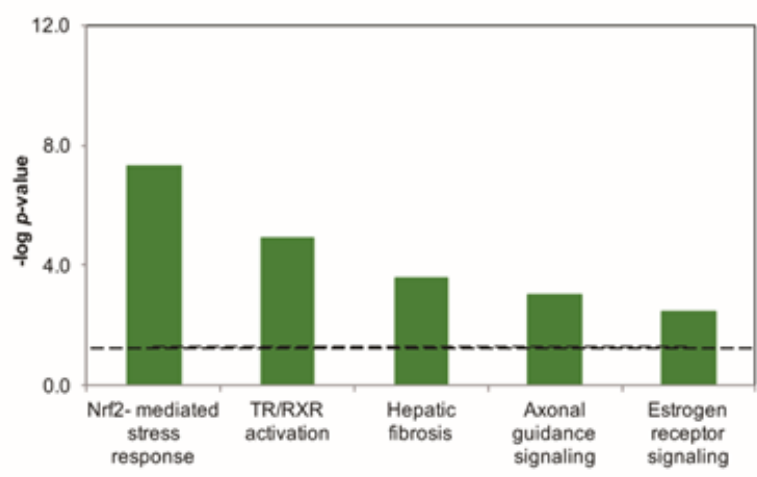

Figure 5. Comparative Analyses of Significantly Modulated Pathways by ITCs in HepG2 and IMR-32 Cell Lines. (A) SFN-treated HepG2 cells; (B) 6-MSITC-treated HepG2 cells; (C) 6-MTITC-treated HepG2 cells; (D) SFN-treated IMR-32 cells (E) 6-MSITC-treated IMR-32 cells; (F) 6-MTITC-treated IMR-32 cells. Dashed line represents the threshold - $\log P$ value 0.05 . Log value $>1.30$ is considered statistically significant.

Pathway Network and Global Functional Analyses by ITCs Stimulation in HepG2 and IMR-32 Cell Lines. To fully extract the essence of huge genomic data from microarray analysis, the significantly modulated genes were subjected to pathway and global functional analyses. Using IPA, the function of cell-specific genes targeted by Japanese wasabiderived ITCs in HepG2 and IMR-32 cell lines would be understood.

The data presented herein indicate that the signaling pathway modulated in HepG2 cells following ITCs treatment is significantly different from the signal pathway modulated in IMR-32 cells (Figure 5). The extent of regulation of the Nrf2-mediated oxidative stress response pathway by ITCs is higher in IMR-32 cells than HepG2 cell. This can be correlated with the higher induction of antioxidants in IMR-32 cells. Genes associated with Nrf2mediated oxidative stress pathway were observed to have different induction levels in HepG2 and IMR-32 cells (Figures 4). Nrf2 is the main transcription factor responsible in the regulation of Nrf2-mediated oxidative stress pathway and it is the master of transcriptional response to oxidative stress. Our results showed that Wasabi-derived ITCs promote activation of $\mathrm{Nrf2}$ even at the protein level (Trio et al., 2016). Nrf2 is widely expressed in the cells. At low level of reactive oxygen species, the nuclear Nrf2 is also present at low amount due to 
the inhibitory function of KEAP1 protein which sequesters Nrf2 in the cytoplasm and target it for proteosomal degradation. However, when the oxidative balance is disrupted, KEAP1 is modified and its ability to target Nrf2 degradation is impaired. Thus, nuclear Nrf2 increases and this increase drives the upregulation of cytoprotective genes (Figure 6). This could also imply that the results could be associated with the cell-specific differences in ARE/ EpRE activation which plays a role in the induction of antioxidant genes (Moehlenkamp et al., 1999). Moreover, detailed evaluation of genes that were involved in transcription activity showed that these genes are transcription cofactors associated with Nrf2 (Table 2). Although both cell types activated Nrf2 in response to ITCs (Figure 6), the cell lines showed differences in the significantly modulated secondary pathways. These may be due to the dissimilarities in the up- and downregulated transcription factors between HepG2 and IMR-32 cells. For instance, gene expression profiles of unstimulated HepG2 showed that Wnt, cell growth and cell survival pathways were deregulated (Adesina et al., 2009). Moreover, our data showed that nuclear factor kappalight-chain-enhancer of activate $\beta$ cells (NFKB), a transcription factor known for its inflammatory and oncogenic functions, is found to be differentially downregulated in HepG2 cells treated with Wasabiderived ITCs but is not activated in IMR-32 cells. This suggests why inflammatory response associated pathways were among the significantly modulated pathways by wasabi-derived ITCs, next to Nrf2 pathway activation in HepG2 cells. Moreover, a high statistical significance for Nrf2 pathway modulation could be attributed to the significant upregulation of genes coding for transcriptional cofactors, $B A C H 1$ and small Maf proteins, in IMR-32 cells. Interestingly, TR/RXR pathway was predominant in IMR-32 cells as a result of ITCs treatment. Activation of transcription factors PPAR/RXR and RAR/RXR have been further explored for neuro therapeutic strategy but studies implicating activation TR/RXR transcription factor remain elusive. Although PPAR/RXR and RAR/ RXR relevance to neurological disease is far from conclusive, studies have already shown promising results. PPARs and RARs display anti-inflammatory effect which could be very useful for pathological processes involving microglia, macrophages and astrocytes and to most neurodegenerative diseases (Neerven et al., 2008). Thus, the results of this study could provide preliminary evidence about the possible neuroprotective effect of ITCs via the TR/ RXR signal transduction. This also implies that TR/ RXR signal transduction could also be a promising target for neuroprotective function and it will be interesting to know the molecular mechanism behind. Whereas in the liver, regulation of RXR

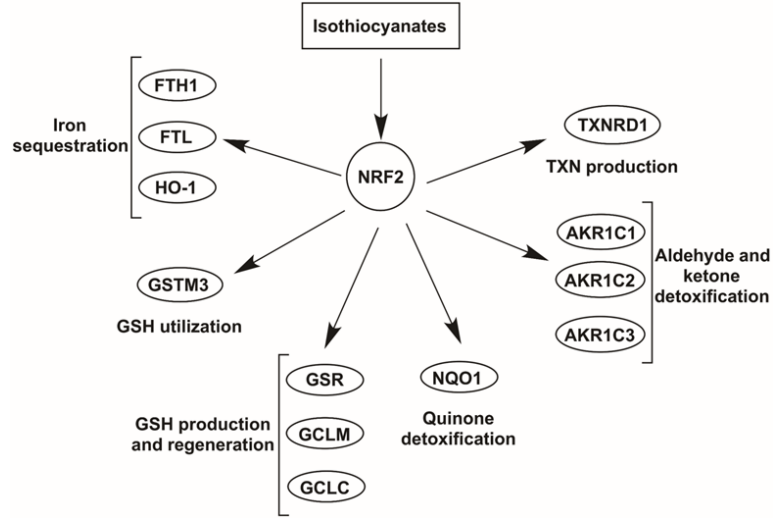

Figure 6. Wasabi-derived isothiocyanates multitargeted pathways. ITCs directly targeted Nrf2-mediated oxidative stress response pathway and indirectly targeted several antioxidant pathways controlled by Nrf2. Genes were group based on their antioxidant functions.

plays an important role in inhibiting apoptosisinduced fragmentation together with transcriptional activities of LXR (Valledor et al., 2004). Thus, this suggests that mechanism of VDR/RXR could be also linked to apoptosis. However, further experiments are needed to support this premise.

\section{CONCLUSIONS}

In conclusion, the results of the gene expression profiling study show that IMR-32 cell line is more sensitive to gene expression changes in response to ITCs treatment than HepG2 cells. Data show that ITCs exhibit varying degree of potency due to its structural differences but share a common primary biological effect in both cell lines. Moreover, all of the three ITCs induced antioxidant-related gene products via regulation of $\mathrm{Nrf2}$ pathway. It should be also noted that ITCs regulate TR/RXR signaling pathway in IMR-32 which need to be further in vivo and in vitro.

\section{REFERENCES}

Adesina AM, Lopez-Terrada D, Wong KK, Gunaratne P, Ngyuyen Y, Pulliam J, Margolin J, and Finegold M: Gene expression profiling reveals signatures characterizing histologic subtypes of hepatoblastoma and global deregulation in cell growth and survival pathways. Hum Pathol 40, 843-853, 2009.

Atwell LL, Beaver LM, Shannon J, Williams DE, Dashwood RH, and Ho E: Epigenetic regulation by sulforaphane: Opportunities for breast and prostate cancer chemoprevention. Curr Pharmacol Rep 1, 102-111, 2015.

Babish JG, Pacioretty LM, Bland JS, Minich DM, 
Hu J, and Tripp ML: Antidiabetic screening of commercial botanical products in 3T3-L1 adipocytes and db/db mice. J Med Food 13, 535-547, 2010.

Chaudhuri D, Orsulic S, and Ashok BT: Antiproliferative activity of sulforaphane in Aktoverexpressing ovarian cancer cells. Mol Cancer Ther 6, 334-345, 2007.

Chen J, Uto T, Tanigawa S, Yamada-Kato T, Fujii M, and Hou DX: Microarray-based determination of anti-inflammatory genes targeted by 6-(methylsulfinyl)hexyl isothiocyanate in macrophages. Exp Ther Med 1, 33-40, 2010.

Chung YK, Or RCH, Lu CH, Ouyang WT, Yang SY, and Chang CC: Sulforaphane down-regulates SKP2 to stabilize p27KIP1 for inducing antiproliferation in human colon adenocarcinoma cells. J Biosci Bioeng 119, 35-42, 2015.

Hou DX, Fukuda M, Fujii $M$, and Fuke Y: Transcriptional regulation of nicotinamide adenine dinucleotide phosphate: quinone oxidoreductase in murine hepatoma cells by 6-(methylsulfinyl) hexyl isothiocyanate, an active principle of wasabi (Eutrema wasabi Maxim.). Cancer Lett 20, 195200, 2000.

Isshiki $\mathrm{K}$ and Tokouka K: Allyl Isothiocyanate and wholesomeness of food. Jpn J Food Microbiol 12, 1-6, 1993.

Itoh K, Chiba T, Takahashi S, Ishii T, Igarashi K, Katoh Y, Oyake T, Hayashi N, Satoh K, Hatayama I, Yamamoto M, and Nabeshima Y: An Nrf2/small Maf heterodimer mediates the induction pf phase II detoxifying enzyme genes through antioxidant response element. Biochem Biophys Res Commun 236, 313-322, 1997.

Keiko F, and Sasahara M: The roles of PDGF in development and during neurogenesis in the normal and diseased nervous system. J Neuroimmune Pharmacol, 9, 168-181, 2014.

Kim YJ, Lee DH, Ahn J, Chung WJ, Kang YJ Seong KS, Moon JH, Ha TY, and Jung $\mathrm{CH}$ : Pharmacokinetics, tissue distribution, and antilipogennic/adipogenic effects of allyl isothiocyanates metabolites. PLOS ONE 10, e0132151, 2015.

Kumagai H, Kashima N, Seki T, Sakurai H, Ishii $\mathrm{K}$, and Ariga T: Analysis of components in essential oil of upland Wasabi and their inhibitory effects on platelet aggregation. Biosci Biotech Biochem 58, 2131-2135, 1994.
Lahiri DK: The stability of beta-amyloid precursor protein in nine different cell types. Biochem $\mathrm{Mol}$ Biol Int 29, 849-858, 1993.

Lee YS: Anti-oxidant effect of Wasabi Japonica extracts. Korean J Oriental Prev Medical Soc 12, 119-126, 2008.

Lemerchant S, Pruvost M, Montaner J, Emery E, Vivien D, Kanninen $\mathrm{K}$, and Koistinaho J: ADAMTS proteoglycanases in the physiological and pathological central nervous system. J Neuroinflammation 10, 133-140, 2013.

Lopez-Terrada D, Gunaratne PH, Adesina AM, Pulliam J, Hoang DM, Nguyen Y, Mistretta TA, Margolin J, and Finegold MJ: Histologic subtypes of hepatoblastoma are characterized by the differential canonical Wnt and Notch patway activation in DLK+ precursors. Hum Path 40, 783-794, 2009.

Mazzarello P: A unifying concept: the history of cell theory. Nat Cell Bio 1, E13-E15, 1999.

Moehlenkamp J, and Johnson JA: Activation of antioxidant/electrophile-responsive elements in IMR-32 human neuroblastoma cells. Arch Biochem Biophys 363, 98-106, 1999.

Myzak MC, Dashwood WM, Orner GA, Ho E, and Dashwood RH: Sulforaphane inhibits histone deacetylase in vivo and suppresses tumorigenesis in Apcmin mice. FASEB J 20, 506-508, 2006.

Neerven SV, Kampmann E, and Mey J: RAR/ RXR and PPAR/RXR signaling in neurological and psychiatric diseases. Prog Neurobiol 85, 433 -451 , 2008.

Nguyen T, Huang HC, and Pickett CB: Transcriptional regulation of the antioxidant response element: Activation by $\mathrm{Nrf} 2$ and repression by MafK. J Biol Chem 275, 15466-15473, 2000.

Okamoto T, Akita N, Nagai M, Hayashi T, and Suzuki K: 6-Methylsulfinylhexyl isothiocyanate modulates endothelial cell function and suppresses leukocyte adhesion. J Nat Med 68, 144-153, 2013.

Ono H, Tesaki S, Tanabe S, and Watanabe M: 6-Methylsulfinylhexyl isothiocyanate and its homologues as food-originated compounds with antibacterial activity against Escherichia coli and Staphylococcus aureus. Biosci Biotechnol Biochem 62, 363-365, 1998.

Ryu HY, Kyung HB, Eun JK, Sang JP, Bong L, and HO YS: Evaluation for the antimicrobial, 
antioxidant and antithrombosis activity of natural spices for fresh cut yam. J Life Sci 17, 652-657, 2007.

Shin IS, Masuda H, and Naohide K: Bactericidal activity of wasabi (Wasabia japonica) against Helicobacter pylori. Int J Food Microbiol 94, 255261, 2004.

Shin SW, Ghimeray AK, and Park CH: Investigation of total phenolic, total flavonoid, antioxidant and allyl isothiocyanate content in the different organs of Wasabi japonica grown in an organic system. Afr J Tradit Complement Altern Med 11, 38-45, 2014.

Sidransky H, Ito N, and Verney E: Influence of alphanaphthyl-isothiocyanate on liver tumorigenesis in rats ingesting ethionine and N-2-flourenylacetamide. J Natl Cancer Inst 37, 677-686, 1966.

Sun CC, Li SJ, Yang CL, Xue RL, Xi YY, Wang L, Zhao QL, and Li DJ: Sulforaphane attenuates muscle inflammation in dystrophin-deficient $\mathrm{mdx}$ mice via NF-E2-related factor 2 (Nrf2)-mediated inhibition of NF- $\kappa \mathrm{B}$ signaling pathway. J Biol Chem 290, 17784-17795, 2015.

Tarozzi A, Angeloni C, Malaguti M, Morroni F, Hrelia S, and Hrelia P: Sulforaphane as a potential protective phytochemical against neurodegenerative diseases. Oxid Med Cell Longev 2013, 415078, 2013.

Trio PZ, Fujisaki S, Tanigawa S, Hisanaga A, Sakao $\mathrm{K}$, Hou DX: DNA microarray highlights Nrf2mediated neuron protection targeted by wasabiderived isothiocyanates. Gene Regul Sys Bio 10, 73-83, 2016.

Trio PZ, Kawahara A, Tanigawa S, Sakao K, Hou DX: DNA microarray profiling highlights Nrf2mediated chemoprevention taegeted by Wasabiderived isothiocyanates in HepG2 cells. Nutr Cancer 69, 105-116, 2017.

Uto T, Fujii M, and Hou DX: Inhibition of lipopolysaccharide-induced cyclooxygenase-2 transcription by 6-(methylsulfinyl) hexyl isothiocyanate, a chemopreventive compound from Wasabia japonical (Miq.) Matsumura, in mouse macrophages. Biochem Pharmacol 70, 1772-1784, 2005a.

Uto T, Fujii M, and Hou DX: 6-(Methylsulfinyl) hexyl isothiocyanate suppresses inducible nitric oxide synthase expression through the inhibition of Janus kinase 2-mediated JNK pathway in lipopolysaccharide-activated murine macrophage.
Biochem Pharmacol 70, 1211-1221, 2005 b.

Uto T, Fujii M, and Hou DX: Effects of 6-(methylsulfinyl)hexyl isothiocyanate on cyclooxygenase-2 expression induced by lipopolysaccharide, interferon- $\gamma$ and 12-O-tetradecanoylphorbol-13-acetate. Oncol Rep 17, 233-238, 2007.

Valledor AF, Hsu LC, Ogawa S, Sawka-Verhelle D, Karin M, and Glass CK: Activation of liver X receptors and retinoid $\mathrm{X}$ receptors prevents bacterialinduced macrophage apoptosis. PNAS 101, 1781317818, 2004.

Vasanthi HR, Mukherjee S, and Das DK: Health benefits of broccoli- a chemico-biological overview. Min Rev Med Chem 9, 749-759, 2009.

Ye L, Yu T, Li Y, Chen B, Zhang J, Wen Z, Zhang B, Zhou X, Li X, Li F, Cao W, and Huang Z: Sulforaphane enhances the ability of human retinal pigment epithelial cell against oxidative stress, and its effect on gene expression profile evaluated by microarray analysis. Oxid Med Cell Longev 2013, 413024, 2013.

Zhang Y, Talalay P, Cho CG, and Posner GH: A major inducer of anticarcinogenic protective enzymes from broccoli: isolation and elucidation of structure. Proc Natl Acad Sci U S A 89, 2399-2403, 1992. 